\title{
Percepción de necesidades en cuidadores familiares de adultos internados en una unidad de cuidados intensivos de una institución prestadora de salud (IPS) privada en Villavicencio, Colombia'
}

\author{
Clara Rocío Galvis López²
}

Emilce Salamanca Ramos 3

doi:10.11144/Javeriana.IE16-2.pncf

Cómo citar: Galvis López CR, Salamanca Ramos E. Percepción de necesidades en cuidadores familiares de adultos internados en una unidad de cuidados intensivos de una institución prestadora de salud (IPS) privada en Villavicencio, Colombia. Investig Enferm. Imagen Desarr. 2014;16(2):81-94. doi:10.11144/Javeriana.IE16-2.pncf

1. Artículo de investigación. Recibido: 26 de marzo de 2014. Revisado: 4 y 16 de abril de 2014. Aprobado: 4 de junio de 2014. Artículo derivado del proyecto de investigación: Percepción de necesidades en cuidadores familiares de adultos internados en Unidad de Cuidados Intensivos de una Institución Prestadora de Salud (IPS) privada, Villavicencio, Colombia, financiado por la Universidad de los Llanos.

2. Enfermera especialista en Salud Ocupacional. Magíster en Enfermeria con énfasis en Cuidado Crónico. Docente, Programa de Enfermeria, Facultad Ciencias de la Salud, Universidad de los Llanos, Villavicencio, Colombia. Integrante Grupo de Cuidado. Correo electrónico: cgalvis@unillanos.edu.co

3. Enfermera. Magíster en Enfermería con énfasis en Salud Familiar. Docente, Programa de Enfermería, Facultad Ciencias de la Salud, Universidad de los Llanos, Villavicencio, Colombia. Correo electrónico: esalamanca@unillanos.edu.co 


\section{Resumen}

Introducción: Ingresar a un familiar en una unidad de cuidados intensivos (UCI) es una experiencia abrumadora para la familia, reconocida como esencial en la salud del paciente. El cuidador, como miembro significativo de la familia, se expone también a situaciones angustiantes que le generan necesidades que se deben identificar para promover su bienestar y calidad de vida. Objetivos: Describir las necesidades del cuidador familiar de pacientes adultos crónicos hospitalizados en UCI en una institución prestadora de salud privada en Villavicencio, Colombia. Material y métodos: Estudio descriptivo, de corte transversal y de tipo cuantitativo. Se utilizó el instrumento Critical Care Family Needs Inventory (CCFNI), de Nancy Molter y Jane Leske, que valora el grado de importancia que los familiares les otorgan a las necesidades de apoyo, comodidad, información, proximidad y seguridad cuando tienen a un familiar ingresado en la UCI. Se entrevistaron cuidadores familiares $(n=37)$ durante un trimestre y se procesó la información en SPSS versión 14. Resultados: La necesidad considerada más importante es la de seguridad, con el $86 \%$; seguida de la de información, con el $82 \%$, y de proximidad, con un $78 \%$. Conclusiones: Para enfermeria, las necesidades sentidas por los cuidadores familiares de adultos en condición de cronicidad hospitalizados en una UCI son base para ofrecer cuidado integral y hacer de la experiencia en UCI momentos menos desalentadores.

Palabras clave: familia; necesidades; cuidadores; cuidado intensivo

\section{Perceived Needs in Family Caregivers of Adults Hospitalized in an Intensive Care Unit of a Private Health Providing Institution in Villavicencio, Colombia}

\section{Abstract}

Introduction: Entering a relative in an intensive care unit (ICU) is an overwhelming experience for the family, recognized as essential to the health of the patient. The caregiver, as a significant member of the family, is also exposed to distressing situations that generate needs to be identified to promote their well-being and quality of life. Objectives: To describe the needs of family caregivers of chronic adult hospitalized patients in ICU in a private health care provider institution in Villavicencio, Colombia. Methods: A descriptive, cross-sectional and quantitative study. It was used The Critical Care Family Needs Inventory (CCFNI) instrument, by Nancy Jane Molter and Leske, which assesses the degree of importance that family give to the support needs, comfort, information, proximity and safety when they have a relative admitted in the ICU. Family caregivers were interviewed $(n=37)$ during a trimester and information on SPSS version 14 was processed. Results: Safety is considered the most important requirement, with $86 \%$; followed by information, with $82 \%$, and proximity, with $78 \%$. Conclusions: For nursing, the felt needs by family caregivers of chronic hospitalized adults in an ICU are the basis to provide comprehensive care and make the experience in ICU less daunting.

Keywords: family; needs; carers; intensive care 


\section{Percepção de necessidades em cuidadores familiares de adultos internados em uma unidade de terapia intensiva de uma provedora de saúde (IPS) privada em Villavicencio, Colômbia}

\section{Resumo}

Introdução: Internar um parente numa unidade de terapia intensiva (UTI) é uma experiência avassaladora para a família, reconhecida como essencial na saúde do paciente. O cuidador, como membro significativo da família, expõe-se também a situações angustiantes que lhe geram necessidades a serem identificadas para promover seu bem-estar e qualidade de vida. Objetivos: Descrever as necessidades do cuidador familiar de pacientes adultos crônicos internados em UTI em uma provedora de saúde privada em Villavicencio, Colômbia. Material e métodos: Estudo descritivo, de corte transversal e tipo quantitativo. Utilizou-se o instrumento Critical Care Family Needs Inventory (CCFNI), de Nancy Molter e Jane Leske, que valora o grau de importância que os familiares outorgam para as necessidades de apoio, comodidade, informação, proximidade e segurança quando têm um familiar interno na UTI. Foram entrevistados cuidadores familiares $(n=37)$ durante um trimestre e a informação foi processada em SPSS versão 14. Resultados: A necessidade considerada mais importante é segurança, com 86\%; seguida de informação, com $82 \%$, e proximidade, com $78 \%$. Conclusões: Para enfermagem, as necessidades sentidas pelos cuidadores familiares de adulto em condição crônica internos em UTI são base para oferecer atendimento integral e fazer da experiência em UTI momentos menos assustadores.

Palavras chave: família; necessidades; cuidadores; atendimento intensivo 


\section{Introducción}

Los indicadores básicos de salud de Colombia, del departamento del Meta y de la ciudad de Villavicencio muestran como primeras causas de morbilidad y mortalidad en adultos maduros y mayores enfermedades crónicas como insuficiencia renal crónica, diabetes mellitus e hipertensión arterial $(1,2)$, y en adultos jóvenes, accidentes de transporte y agresiones.

Cuando una persona padece alteraciones crónicas y su estado de salud se deteriora, requiere atención especializada en una unidad de cuidado intensivo (UCI) para atender las alteraciones de los diferentes sistemas y conseguir un mejor pronóstico (3). Allí se encuentran los cuidadores (4), aquellas personas que los atienden en el domicilio de forma continua y durante periodos prolongados (5) y con presencia esencial para la recuperación de la salud de su familiar $(6,7)$.

El ambiente de UCI es de gran complejidad, por los equipos de alta tecnología, el personal de salud calificado y las terapéuticas de salud que se ofrecen, acordes a los últimos avances y evidencias científicas, al tiempo que se siguen protocolos para el cuidado seguro y de calidad. Dichos escenarios tienen implicaciones en la persona ingresada, en la familia como la unidad de cuidado (8) y en el cuidador, sobre todo cuando no se le involucra como parte del cuidado integral.

La experiencia de tener a un familiar ingresado en una UCI expone al cuidador a un mundo para la mayoría desconocido, lleno de expectativas. Supone nuevas responsabilidades de cara al cuidado de su familiar y le genera, además de la preocupación por la salud de su ser querido, ansiedad, estrés, aislamiento $(8,9)$, soledad y ajustes diarios en su cotidianidad. "La hospitalización de un familiar en una UCI afecta psicológica y socialmente tanto al paciente como a sus familiares, quienes suelen experimentar estrés, desorganización y sentimientos de desamparo" (6); así como cólera, culpa, desesperación, ansiedad y miedo (7), y requieren "cuidado y atención oportuna" (10).

En las UCI existen diversos actores: el paciente, el personal de salud y el cuidador familiar (11). Este último cada día tiene más protagonismo, porque ha cuidado en el hogar a su familiar. El profesional de enfermería desempeña un rol primordial, pues, además de ser responsable del cuidado directo del paciente, está llamado a proporcionar cuidado a sus familias, atendiendo sus principales necesidades $(8,11)$ comprendiendo que la necesidad para un ser humano es "la sensación de carencia de algo unida al deseo de satisfacerla" (12).

Con este estudio se buscó explorar las percepciones sobre el grado de importancia que le otorgan a sus propias necesidades los cuidadores familiares de adultos internados en la UCI de una institución prestadora de servicios de salud (IPS) privada de Villavicencio, Colombia.

\section{Materiales y métodos}


cuidadores familiares de pacientes adultos que padecen alteraciones crónicas de salud y que están hospitalizados en la UCI (n = 37) de una IPS privada ubicada en Villavicencio, Colombia, durante un trimestre y que cumplieran los siguientes criterios de inclusión:

- Tener edad igual o superior a 18 años.

- Tener un familiar adulto con alteración crónica de salud hospitalizado en la UCI como mínimo 24 horas.

- Haber cuidado al paciente crónico en el mes anterior a la hospitalización.

- Haber visitado al paciente crónico hospitalizado en UCI, por lo menos, una vez durante el periodo de hospitalización.

- Estar de acuerdo con participar del estudio, firmando un consentimiento informado.

Durante el periodo en que se recoge la muestra dos de los cuidadores familiares no accedieron a conceder la entrevista por razones de carácter personal.

Se utilizó el Inventario de Necesidades de Familia de Cuidados Críticos (CCFNI, por su sigla en inglés). La fiabilidad de consistencia interna y validez de constructo del CCNFI lo realizó Leske, por medio del estudio con 677 sujetos, recogidos por 21 investigadores de enfermería en 14 estados durante 9 años (1980-1988). El coeficiente de consistencia interna alfa del total CCFNI fue de 0,92 (13). Escala creciente de 1 a 4, tipo Likert, con cuatro alternativas de respuesta: 1 (no es importante), 2 (poco importante), 3 (importante) y 4 (muy importante). Se midió así el grado de importancia que los familiares otorgan a las necesidades de seguridad, apoyo, información, comodidad y proximidad (14).

Se cursa solicitud personal a la Dra. Jane Leske, quien autorizó el uso del CCFNI y remite las versiones en inglés y español del instrumento y medidas psicométricas citadas. Se realizó una prueba piloto en 10 cuidadores familiares de personas con alteraciones crónicas en una UCI de una IPS privada de la ciudad de Villavicencio, diferente a la IPS donde se realizó el estudio. Para la recolección y análisis de la información se llevó a cabo el reconocimiento de los familiares que cumplieran los criterios de inclusión, a través de la revisión de los registros de visita, del kárdex de enfermería, de historia clínicas o por entrevista en la sala de espera. Posteriormente se les invitó a participar del estudio, explicándoles el objetivo y solicitando su autorización y firma del consentimiento informado. Las entrevistas se realizaron en un espacio reservado, independiente de la UCI, luego de la visita o en el domicilio, a solicitud del cuidador familiar.

La información se procesó con el paquete estadístico Statistical Program for Social Sciences (SPSS) versión 14.0 para Windows, con las 45 declaraciones que comprende el CCFNI. Se determinaron la media, la desviación estándar y el coeficiente de variación para cada una de las 45 afirmaciones que componen las subescalas del CCFNI. Finalmente, se estimó la confiablidad del instrumento para valorar las necesidades de los cuidadores familiares en UCI a través del alfa de Conbrach, que reportó 0,88. En cumplimiento 
con lo establecido con los requerimientos éticos, se tuvo presente lo expuesto en la Resolución 008430 de 1993, del Ministerio de Salud de Colombia, y la autorización por parte de las directivas de las instituciones salud.

\section{Resultados}

La tabla 1 indica el perfil del paciente y del cuidador familiar. El perfil del cuidador corresponde a mujeres, en la mayoría de los casos. El perfil de los pacientes en situación de cronicidad hospitalizados se ve más en hombres con relación a las mujeres.

TABLA 1. Perfil de pacientes hospitalizados en UCI y los cuidadores de la IPS privada de la ciudad de Villavicencio, Colombia (2012)

\begin{tabular}{|l|l|}
\hline \multicolumn{1}{|c|}{ Perfil del paciente (n = 37) } & \multicolumn{1}{c|}{ Perfil de cuidador (n = 37) } \\
\hline Adultos mayores & Sexo: mujer \\
\hline Nivel de estudios: primarios & Nivel de estudios: primarios \\
\hline Situación laboral: hogar y pensionados & Relación de parentesco: hija, esposa \\
\hline $\begin{array}{l}\text { Antecedentes de hipertensión arterial y } \\
\text { diabetes tipo II y enfermedad pulmonar } \\
\text { obstructiva crónica }(72 \%)\end{array}$ & $\begin{array}{l}24 \% \text { y } 29 \% \text { le dedican entre } 1 \text { y } 5 \text { años } \\
\text { yespectivamente en el domicilio }\end{array}$ \\
\hline $\begin{array}{l}\text { Diagnóstico de ingreso: infarto agudo } \\
\text { al miocardio y enfermedad coronaria } \\
\text { severa }\end{array}$ & $\begin{array}{l}32 \% \text { no tienen experiencias anteriores } \\
\text { de hospitalizaciones de su familiar en } \\
\text { UCI }\end{array}$ \\
\hline
\end{tabular}

Fuente: Elaboración propia

Los resultados (tabla 2) indican que el mayor grado de importancia se encuentra en las necesidades de seguridad (86\%) e información (82\%), con un coeficiente de variación que reporta mayor homogeneidad, además de los promedios más altos, seguido de proximidad (78\%), luego apoyo (57\%) y confort (54\%).

TABla 2. Estadistica descriptiva de las puntuaciones de las subescalas ( $\mathrm{n}=37$ ), de la IPS privada de la ciudad de Villavicencio, Colombia (2012)

\begin{tabular}{|l|c|c|c|c|}
\hline Dimensiones & $\%$ & Media & $\begin{array}{c}\text { Desviación } \\
\text { estándar }\end{array}$ & $\begin{array}{c}\text { Coeficiente } \\
\text { de variación }\end{array}$ \\
\hline $\begin{array}{l}\text { Seguridad } \\
(\mathrm{A})^{*}\end{array}$ & 86 & 3,85 & 0,07 & 1,76 \\
\hline $\begin{array}{l}\text { Información } \\
(\mathrm{I})^{*}\end{array}$ & 82 & 3,79 & 0,10 & 2,74 \\
\hline $\begin{array}{l}\text { Proximidad } \\
(\mathrm{P})^{*}\end{array}$ & 78 & 3,65 & 0,34 & 9,46 \\
\hline Apoyo $(\mathrm{S})^{*}$ & 57 & 3,33 & 0,37 & 11,3 \\
\hline Confort $(\mathrm{C})^{*}$ & 54 & 3,26 & 0,30 & 9,33 \\
\hline
\end{tabular}

* Iniciales en inglés

Fuente: Elaboración propia 
La tabla 3 indica diez necesidades de los cuidadores familiares con mayor grado de importancia, y la tabla 4, diez con menor grado de importancia. Aun cuando la necesidad de seguridad, vista de manera global, es considerada de mayor grado de importancia por los cuidadores, discriminadas las afirmaciones por cada dimensión de las necesidades, 4 de las 10 necesidades reportadas como importantes y muy importantes son componentes de la necesidad de proximidad.

TABLA 3. Diez necesidades más importantes según las afirmaciones CCFNI identificadas por los cuidadores familiares $(n=37)$

\begin{tabular}{|c|c|c|c|c|}
\hline Ítem & Afirmación & Media & $\begin{array}{l}\text { Desvia- } \\
\text { ción } \\
\text { estándar }\end{array}$ & $\begin{array}{c}\text { Coefi- } \\
\text { ciente de } \\
\text { variación } \\
(\%)\end{array}$ \\
\hline 39 & $\begin{array}{l}\text { Informen a tiempo cuando se traslada } \\
\text { el paciente }(\mathrm{P})\end{array}$ & 3,97 & 0,164 & 4 \\
\hline 40 & $\begin{array}{l}\text { Que se le llame a la casa cuando haya } \\
\text { cambios en la condición del paciente (P) }\end{array}$ & 3,95 & 0,229 & 6 \\
\hline 41 & $\begin{array}{l}\text { Recibir información sobre el paciente } \\
\text { al menos una vez al día }(\mathrm{P})\end{array}$ & 3,95 & 0,229 & 6 \\
\hline 3 & Hablar con el médico todos los días (I) & 3,95 & 0,229 & 6 \\
\hline 36 & $\begin{array}{l}\text { Que las horas de visita comiencen } \\
\text { puntualmente (P) }\end{array}$ & 3,95 & 0,229 & 6 \\
\hline 1 & Conocer el pronóstico del paciente (A) & 3,92 & 0,277 & 7 \\
\hline 5 & $\begin{array}{l}\text { Que las preguntas sean contestadas } \\
\text { con honestidad (A) }\end{array}$ & 3,92 & 0,277 & 7 \\
\hline 16 & $\begin{array}{l}\text { Conocer el tratamiento que se le está } \\
\text { proveyendo al paciente (I) }\end{array}$ & 3,92 & 0,277 & 7 \\
\hline 42 & $\begin{array}{l}\text { Sentir que los empleados del hospital } \\
\text { se preocupan por el paciente (A) }\end{array}$ & 3,89 & 0,315 & 8 \\
\hline 2 & $\begin{array}{l}\text { Ser orientado sobre el ambiente de la } \\
\text { UCI antes de entrar por primera vez (S) }\end{array}$ & 3,81 & 0,397 & 10 \\
\hline
\end{tabular}

Fuente: Elaboración propia

Se encontró que la necesidad de que "le informen a tiempo cuando se traslada a su familiar" fue percibida como la más crucial, y la necesidad de "estar solo en algún momento" fue categorizada como de menor importancia por el cuidador. 
TABla 4. Diez necesidades menos importantes según las afirmaciones CCFNI identificadas por los cuidadores familiares $(n=37)$

\begin{tabular}{|c|l|c|c|c|}
\hline Ítem & \multicolumn{1}{|c|}{ Afirmación } & Media & $\begin{array}{c}\text { Desvia- } \\
\text { ción } \\
\text { estándar }\end{array}$ & $\begin{array}{c}\text { Coefi- } \\
\text { ciente de } \\
\text { variación } \\
\text { (\%) }\end{array}$ \\
\hline 10 & Estar solo en algún momento (S) & 2,51 & 1,096 & 44 \\
\hline 18 & $\begin{array}{l}\text { Tener un lugar donde estar solo } \\
\text { mientras esté en el hospital (S) }\end{array}$ & 2,65 & 1,116 & 42 \\
\hline 8 & $\begin{array}{l}\text { Tener buena comida disponible en el } \\
\text { hospital (C) }\end{array}$ & 2,68 & 1,132 & 42 \\
\hline 20 & $\begin{array}{l}\text { Tener muebles cómodos en el área } \\
\text { de espera (C) }\end{array}$ & 3,03 & 1,190 & 39 \\
\hline 29 & $\begin{array}{l}\text { Hablar con la misma enfermera } \\
\text { todos los dias (P) }\end{array}$ & 2,97 & 1,142 & 38 \\
\hline 10 & $\begin{array}{l}\text { Poder visitar al paciente a cualquier } \\
\text { hora (P) }\end{array}$ & 3,30 & 1,051 & 32 \\
\hline 37 & $\begin{array}{l}\text { Que se le oriente en los servicios de } \\
\text { capellanía (S) }\end{array}$ & 3,08 & 0,983 & 32 \\
\hline 30 & Sentir libertad de llorar (S) & 3,05 & 1,040 & 30 \\
\hline 7 & $\begin{array}{l}\text { Que me permitan expresar mis senti- } \\
\text { mientos sobre lo que está pasando (S) }\end{array}$ & 3,30 & 0,968 & 29 \\
\hline 45 & $\begin{array}{l}\text { Tener el área de espera cerca del } \\
\text { paciente (P) }\end{array}$ & 3,35 & 0,978 & 29 \\
\hline
\end{tabular}

FUente: Elaboración propia

\section{Discusión}

Las características sociodemográficas se muestran similares a las reportadas en otros estudios sobre el perfil de los pacientes hospitalizados en una UCI y concuerdan en variables como edad, sexo y experiencias previas en UCI. La morbilidad es coincidente con las reportadas en diferentes indicadores de salud $(1,2)$ y son motivo de ingresos o reingresos a la UCI. Los hallazgos señalan que, en la mayoría de los casos, los pacientes son de mayor edad que los cuidadores (15): esposos e hijos, por ejemplo. Similar situación ocurre con el perfil de los cuidadores con respecto a edad, sexo y ocupación (16), pero se presentan diferencias en el nivel de escolaridad (16). Se resalta que una tercera parte de los cuidadores ya han tenido experiencias con hospitalizaciones anteriores de su familiar en UCI (17), vivencias que pueden variar su percepción.

Hay concordancia en la necesidad de información; sin embargo, necesidades como "esperanza, tener salas de espera cerca de las UCI, disponer de lugares para estar solos y poder visitar a su ser querido a cualquier hora" (8), a diferencia de Molter, no son muy o importantes para los cuidadores del estudio. Incluso no manifiestan como necesidad sentida la "ayuda con los problemas financieros" (8), reportados por la autora del 
CCFNI. En cuanto a las necesidades consideradas poco o no importantes, también hay similitudes "en tener un lugar para estar solo" mientras está en la institución de salud y "ser informados sobre servicios de capellanía" (17). En la mayoría de los estudios, los cuidadores encuentran que la información es, de las necesidades, la más sentida y se encuentra relacionada con mayor grado de satisfacción, en la medida en que en las UCI se entregue material educativo como apoyo a la información clínica de su familiar hospitalizado (18).

Los familiares que tienen necesidad de información suelen querer "que se les informe a tiempo cuando se traslada al paciente, que se les llame a casa cuando haya cambios en la condición de su ser querido, recibir por lo menos una vez al día información sobre su familiar, hablar con el médico todos los días y conocer el tratamiento que se le está ofreciendo a su familiar hospitalizado". Tales necesidades sentidas coinciden con las reportadas en la revisión de literatura expuesta por Pardavila Belio y Vivar, en el 2012 (8).

Teniendo en cuenta que la proximidad se busca a través del apego cuando un adulto está expuesto a condiciones adversas (19), en el caso de los cuidadores se evidencian necesidades consideradas "manifestaciones de proximidad" como: "Informen a tiempo cuando se traslada el paciente": que se les llame a la casa cuando su familiar presenta cambios en condición de salud, que por lo menos se reciba información sobre la situación de salud de su familiar por lo menos una vez al día y que cuando se encuentre dentro de la institución las horas de visita empiecen puntuales. Es importante mencionar que el cuidador ha establecido un apego natural con su familiar, por su relación permanente al dar el cuidado en su domicilio; cercanía que también requiere satisfacer en la hospitalización de su familiar en una UCI.

Es conocido que muchas emociones de los familiares están relacionadas no solo con la estancia del ser querido en las salas de UCI, sino que pueden también en los traslados (20). Alteraciones como ansiedad y depresión son reportadas cuando al familiar se le da de alta para otro servicio o cuando fallece. El traslado puede ser visto como positivo o negativo (20): el sentir miedo a lo que está por venir, verse contrariado, olvidar la información recibida de manera verbal son también sentimientos y conductas expresadas por cuidadores (21).

Si se tiene en cuenta que los cuidadores necesitan "conocer la terapéutica instaurada a su ser querido y conocer el pronóstico" de su familiar para satisfacer su necesidad de información, las preocupaciones por el "pronóstico de la condición crítica de su familiar y el inicio puntual de las horas de visitas" pueden impactar en la salud del cuidador familiar e incrementar sentimientos y conductas de estrés, desamparo, confusión, preocupación, irritabilidad, depresión e incertidumbre (22-24). Que el "personal que labora en la institución se preocupe por su familiar y que las preguntas sean contestadas con honestidad", proporcionan seguridad al cuidador, y ello coincide con lo reportado en la literatura (15). Cuando el familiar no recibe información del personal de la UCI, se ve enfrentado a 
recibirla de manera incompleta, no correcta e incluso haciendo inferencias equivocadas de las expresiones faciales o frases sueltas del personal que lo atiende (23).

Aun cuando algunos cuidadores han tenido experiencias anteriores de hospitalizaciones de su familiar en una UCI y supone haber recibido orientación para la permanencia en esta, sienten de todas maneras la necesidad de apoyo, al requerir del personal asistencial, ser "orientados sobre el ambiente de la unidad antes de entrar por primera vez", probablemente porque que cada experiencia de hospitalización en UCI es diferente, es individual y es única según las investigadoras. La vivencias para los cuidadores que por primera vez entran a las salas de UCI genera expectativa (24), impresión y asombro por percibir olores característicos de medicamentos, alarmas de los monitores, paredes blancas y ambiente de tensión y de zozobra, que evidencia la necesidad de que el cuidador sea orientado en el ambiente de la UCI antes de entrar por primera vez.

Los resultados mostraron que las diez necesidades que son poco o no son importantes para los cuidadores familiares tienen que ver con "querer estar solo, tener un lugar para sentir libertad de llorar, que se le oriente a través de servicios religiosos y que se le permita expresar los sentimientos sobre lo que está pasando"; necesidades que comprenden la dimensión de apoyo. Las investigadoras suponen que se desprenden de sus propios sentimientos y centran toda su atención hacia el estado, evolución y pronóstico de la persona, contrario a lo reportado en otros estudios, donde la privacidad y el recogimiento sí son necesidades referidas como significativas por otros cuidadores (22).

Así, los cuidadores no perciban como importante que sea "la misma profesional de enfermería con la que pueda hablar todos los días, que le permitan expresar sus sentimientos sobre lo que está sucediendo y que se le oriente en servicios de capellanía", la identificación y satisfacción de estas necesidades le proporcionan cercanía, fortaleza (3), tranquilidad, paz e incluso confianza para compartir sentimientos en beneficio de su bienestar y calidad de vida. Expresar "dudas y sentimientos a los enfermero(a) les hace sentir confianza, aumentar lo que saben de la condición de su familiar y los tranquiliza", pues consideran esencial la información en los procesos de acompañamiento al cuidador que tienen pacientes internados en una UCI (25).

Los resultados de este estudio discrepan de hallazgos reportados en la literatura, en los cuales la posibilidad de visitar con más frecuencia a su familiar es muy significativo (26). Los resultados demostraron que los cuidadores familiares "no consideran importante visitar a su familiar a cualquier hora", y ello se puede suponer por lo arraigado que se encuentra en las personas que los horarios no son flexibles y que las visitas tienen restricciones. Se reconocen diferentes tipos de apoyo que se pueden dar en las interacciones entre profesionales de la salud, la familia - como la red de apoyo primario- (3), los cuidadores y los allegados durante la estancia de un paciente en UCI. El apoyo se perdería si los profesionales de enfer- 
mería no los identifican y los retoman como significativos en los momentos del encuentro familiar en las visitas.

Necesidades prácticas (8) como tener "buena comida disponible, muebles cómodos y un área de espera cerca del paciente”, consideradas de menor importancia, acrecientan el riesgo que trae consigo el cuidador. E1 ingreso de un ser querido a la UCI, además de ocasionar gran ansiedad y depresión, incrementa el riesgo de no atender necesidades básicas y la posibilidad de que se asuman conductas poco saludables como falta de descanso, escasa actividad física y dieta no balanceada $(24,27)$. Los hallazgos difieren de la necesidad de comodidad expresada por cuidadores que consideran importantes el espacio físico y muebles cómodos, mayor confort y privacidad de la salas de espera que existen en las UCI (26) e, incluso, que las salas de espera se trasladen a un lugar más cercano (22). El confort y la cercanía que se genera en las salas de espera siempre son importantes para los cuidadores (10), y cuando la sala de espera se encuentra lejos de la entrada de la UCI, ocasiona en los cuidadores sentimientos de inseguridad (28). En general, en el estudio hay coincidencias y diferencias en las necesidades identificadas como importantes y no importantes, según la revisión de literatura expuesta en el estudio, que presenta una síntesis de 34 estudios en familias realizados desde 1979 al 2011 (10).

\section{Conclusiones}

Las dimensiones de necesidades de seguridad, información y proximidad son percibidas como de mayor grado de importancia en los cuidadores familiares de pacientes con alteraciones crónicas hospitalizados en la UCI. Los resultados ponen de manifiesto que hay coincidencia en la priorización de necesidades, como que se informe cuando se traslada el paciente, seguida de la necesidad del llamado cuando sucedan cambios en las condiciones de la salud. Otras necesidades muy importantes son recibir información por lo menos una vez al día y que las horas de visita empiecen a tiempo. De poca importancia para los cuidadores, pero de sumo interés para los profesionales de enfermería, se destaca tener el área de espera cerca del paciente, disponer de comida saludable y muebles cómodos en el área de espera.

Aunque la investigación se ejecutó en una IPS de carácter privado, donde se atiende a familiares que proceden de otros servicios de la misma IPS o de otras IPS, los resultados no se pueden generalizar; sin embargo, presentan una panorámica para establecer a corto y mediano plazos intervenciones dirigidas al cuidado integral del cuidador familiar: la satisfacción de necesidades por medio de material de apoyo educativo, la formación en cuidado humanizado al recurso humano a través de procesos educativos y algunos cambios organizativos y locativos, que se consideran de vital importancia.

El reconocimiento y la inserción de la familia de los pacientes plantean cambios fundamentales tanto para las instituciones de salud como para las prácticas de cuidado de enfermería, a fin de planificar la atención 
de los cuidadores, a partir de sus realidades, sentimientos y experiencias en un contexto de totalidad.

\section{Referencias}

1. Ministerio de Salud. Situación de salud en Colombia: indicadores básicos de salud 2011 [internet]. Bogotá; 2012 [citado 2013 jun 3]. Disponible en: http://www.minsalud.gov.co/salud/paginas/indicadoresbasicossp.aspx.

2. Secretaría Seccional de Salud del Meta. Análisis de la situación de salud departamento del Meta 2011 [internet]. 2011 [citado 2013 jun 3]. Disponible en: http://www.minsalud.gov.co/plandecenal/mapa/ Analisis-de-Situacion-Salud-META-2011.pdf.

3. Márquez Herrera M. La experiencia del familiar de la persona hospitalizada en la unidad de cuidados intensivos [internet]. Bogotá; 2012 [tesis], Universidad Nacional de Colombia [citado 2014 may 11]. Disponible en: http://www.bdigital.unal.edu.co/view/divisions/ fac $=5$ Fenf $/ 2012 . h t m l$.

4. Benito Domingo ML, García Manzano AI, López Sagospe I, Benito RM, Peláez Ortiz R, Rojas Cabrera A. Detección de necesidades de los familiares de pacientes ingresados en la Unidad de Ictus. Enferm Neurol. 2012 may;35(1):6-11.

5. Crespo López M, López Martínez J. El estrés en cuidadores de mayores dependientes. Madrid: Pirámide; 2007.

6. Gómez Carretero P, Soriano Pastor JF, Monsalve Dolz V, Andrés Ibáñez JD. Satisfacción con la información: posible variable interviniente en el estado de ánimo de cuidadores primarios de pacientes críticos. Clínica y Salud. 2009;20(1):91-105.

7. Bernat R. Necesidades de la familia del paciente crítico, revisión bibliográfica. Nursing. 2008;26(9):60-3.

8. Pardavila Belio MI, Vivar CG. Necesidades de la familia en las unidades de cuidados intensivos: revisión de literatura. Enferm Intensiva. 2012;23(2):51-67.

9. Solano Ruiz MC, Siles González J. Análisis de las vivencias de un paciente en cuidados intensivos desde la perspectiva de la complejidad. Cul Cuid. 2003;(14):83-91.

10. Jiménez-Ocampo VF, Zapata-Gutiérrez LS, Diaz-Suárez L. Capacidad de afrontamiento y adaptación de los familiares del paciente adulto hospitalizado en una unidad de cuidado intensivo. Aquichán. 2013;13(2):159-72.

11. Pallares Martí AP. El mundo de las unidades de cuidados intensivos: la última frontera [internet]. Tarragona; 2013 [tesis], Universitat Rovira I Virgili [citado 2013 jun 3]. Disponible en: http://www.tesisenred.net/bitstream/handle/10803/8436/Pallares.pdf?sequence $=1$. 
12. Elizalde A, Martí Vilar M, Martínez Salvá F. Una revisión crítica sobre las necesidades humanas desde el enfoque centrado en la persona. Polis. 2006:5(15).

13. Leske JS. Internal psychometric properties of the critical care family needs inventory. Heart Lung. 1991 May;20(3):236-44.

14. Leske JS. Comparison ratings of need importance after critical illness from family members with varied demographic characteristics. Am Crítico Nursing Care. 1992 Dec;4(4):607-13.

15. Vega Angarita OM. Percepción del apoyo social funcional en cuidadores familiares de enfermos crónicos. Aquichán; 2011 Dic;11(3):274-86.

16. García Navarro S, Fernández Vázquez M, Cumbrera Díaz EM, Morano Torrescusa MJ, Alfonso Pérez D, Contreras Pereira I. Cuidando a la familia en UCI. Biblioteca Lascasas [internet]. 2007 [citado 2013 jun 5];3(2):1-24. Disponible en http://www.index-f.com/lascasas/ documentos/1c0248.php.

17. Freitas KS, Kimura M, Azevedo K. Necesidades de los familiares en unidades de terapia intensiva: análisis comparativo entre hospital público y privado. Rev Latino-Am Enfermagem [internet]. 2007 enero-febrero [citado 2013 jun 2];15(1):84-92. Disponible en: http:// www.scielo.br/pdf/rlae/v15n1/es_v15n1a13.pdf.

18. Campo Martínez MC, Cotrina Gamboa MJ. Relación de ayuda al familiar del paciente en situación crítica. Enferm Glob [internet]. 2011 Oct [citado 2013 jun 11];10(24):103-9. Disponible en: http://scielo.isciii.es/scielo.php?pid=S1695-61412011000400009\&script $=$ sci arttext.

19. Azoulay E, Sprung CL. Family-physician interactions in the intensive care unit. Crit Care Med. 2004 Nov;32(11):2323-8.

20. Torrents R, Torras O, Saucedo Fernández MJ, Surroca Sales L, Jover Sancho C. Impacto de los familiares del paciente crítico: ante una acogida protocolizada. Enferm Intensiva. 2003;14(2):49-61.

21. Achury DM, Achry LF, Ramírez AM. La familia y las visitas en las unidades de cuidado intensivo. The World of Critical Care Nursing [internet]. 2011 [citado 2013 jun 5];8(1):1-8. Disponible en: http:// connectpublishing.org/assets/journals/8_1_1.pdf.

22. Albistur MC de, Bacigalupo JC, Gerez J, Uzat M, Ebeid A, Maciel M et al. La familia del paciente internado en la Unidad de Cuidados Intensivos. Rev Med Uruguay. 2000;16:243-56.

23. Cerón Polanco YM. Aspectos presentes en la comunicación de la enfermera, la familia y la institución en la Unidad de Cuidado Intensivo [internet]. Bogotá; 2008 [tesis], Pontificia Universidad Javeriana [citado 2013 jun 5]. Disponible en: http://www.javeriana.edu. co/biblos/tesis/enfermeria/tesis52.pdf.

24. Gómez-Carretero P, Monsalve V, Soriano JF, de Andrés J. E1 ingreso en la unidad de cuidados intensivos la repercusión en el familiar del paciente. Boletín de Psicología. 2006;87:61-87. 
25. Alfonso Pérez DP, Fernández Vásquez M, García Navarro S, Contreras Pereira I, Cumbreras Díaz EM, Marín Sánchez B. Información enfermera: satisfacción de los familiares de pacientes en cuidados críticos. Biblioteca Lascasas [internet]. 2007 [citado 2013 may 11];3(1). Disponible en: http://www.index-f.com/lascasas/documentos/lc0215.pdf.

26. Maruiti MR, Galdeano LE. Necesidades de los familiares de pacientes ingresados en la unidad de cuidados intensivos. Acta Paul Enferm [internet]. 2007 mar. [citado 2013 jun 5]; 20(1):37-43. Disponible en: http://www.scielo.br/scielo.php?script=sci_arttext\&pid $=$ S0103-21002007000100007.

27. Gómez Martínez S, Ballester Arnal R, Gil Juliá B. El Cuestionario de Necesidades de los Familiares de Pacientes de Cuidados Intensivos (CCFNI) versión breve: adaptación y validación en población española. An Sist Sanit Navar. 2011 Dic;34(3):349-61.

28. Santana Cabrera L, Sánchez Palacios M, Hernández Medina E, García Martul M, Eugenio Ronaina P, Villanueva Ortiz Á. Necesidades de los familiares de pacientes de cuidados intensivos: percepción de los familiares y del profesional. Med. Intensiva. 2007 sep;31(6):273-80. 